\title{
Mediación en el sistema público de salud: oportunidades para mejorar
}

\author{
DENISSE LAGOS TISSIE ${ }^{1,2, a}$, LIDIA BRAVO R. 3,a
}

\section{Mediation in public healthcare: opportunities for improvement}

Background: Mediation in healthcare is a non-adversarial process to resolve a dispute risen between patients and health providers during medical attention Aim: To characterize the mediation process taking place in the public health system in Chile, from its start until 2017. Material and Methods: Cross-sectional descriptive study. Under the Transparency Law, information about mediation processes between 2005 and 2017 was requested to the State Defense Council (CDE in its Spanish acronym). This data was complemented with the information available on the website of this agency. Results: Ninety four percent of the complaints filed at the CDE were deemed eligible for mediation. Only 19\% of the concluded cases led to an agreement between the disputing parties. The agreements reached were mostly monetary compensation, medical assistance, and apologies/explanation of the facts. The average amount of compensation reached $\$ 14,862,088$ (Chilean pesos). The most commonly claimed damage resulting from medical care was partial disability. The medical specialties more often claimed were Obstetrics and Gynecology, General Surgery, and Internal Medicine. Conclusions: The analysis of conducted mediations is a source of feedback for healthcare staff and health institutions. It would greatly contribute to prevent possible damage and medical conflicts, specially within the specialties with the most complaints. Improvements to the existing legislation are required to ensure free access for all the population.

(Rev Med Chile 2020; 148: 211-215)

Key words: Compensation and Redress; Defensive Medicine; Malpractice; Negotiating.

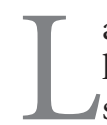

a Organización Mundial de la Salud define la seguridad de los pacientes como "la ausencia (...) de daño innecesario o potencial asociado a la atención sanitaria”. Es un principio básico que esta atención no cause más daño que la enfermedad, coincidiendo con el principio bioético de no maleficencia ${ }^{1,2}$.

Entenderemos como "daño asociado a la atención sanitaria” a cualquier alteración del organismo que deriva de las medidas adoptadas durante la prestación clínica, y no el que se debe a una enfermedad o lesión subyacente. Estos daños suelen ser fuente de conflicto entre el afectado y el equipo de salud, siendo potencial fuente de un enfrentamiento judicial ${ }^{1,3}$.

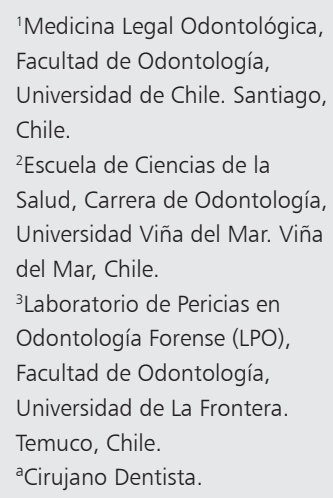

Trabajo no recibió financiamiento. Los autores declaran no tener conflictos de interés.

Recibido el 9 de julio de 2019, aceptado el 2 de diciembre de 2019

Correspondencia a:

Lidia Bravo Rodríguez

Avenida Francisco Salazar 01145. Temuco, Chile.

lidiabravo@ufrontera.cl
En Chile, la mediación por daños en salud fue consagrada en la Ley No19.966, cuyo propósito fue desjudicializar los conflictos sanitarios, estableciendo una instancia de mediación previa al ejercicio de acciones judiciales civiles contra un prestador de salud. La mediación es un procedimiento no adversarial que tiene como objetivo que, mediante la comunicación directa entre las partes y, con intervención de un mediador, estas lleguen a un acuerdo extrajudicial ante una controversia. Cuando la prestación de salud que origina el reclamo fuera otorgada por prestadores privados, la mediación debe realizarse ante mediador inscrito en la Superintendencia de Salud (SIS), quien recibe un pago de las partes, mientras 
que las reclamaciones por atenciones con prestadores públicos, deben mediarse ante el Consejo de Defensa del Estado (CDE), en forma gratuita, voluntaria, confidencial e imparcial ${ }^{4,5}$.

El conflicto sanitario de competencia del CDE comprende a los prestadores institucionales públicos que forman parte de las redes asistenciales o sus funcionarios, además de los establecimientos privados que suscriban convenio con el servicio de salud respectivo. Se excluyen del proceso de mediación ante el $\mathrm{CDE}^{6,7}$ :

- Médicos atendiendo de forma particular mediante convenio en hospitales públicos.

- Consultorios dependientes de corporaciones (personas jurídicas de derecho privado).

- Hospital Clínico de la Universidad de Chile, y hospitales de Fuerzas Armadas, de Orden y Penitenciarios, por no pertenecer a la red asistencial.

El objetivo del presente estudio es caracterizar el proceso de mediación por daños en salud en el sector público desde su implementación, el año 2005, hasta el año 2017, con el fin de exponer sus principales características y resultados y discutir algunas de sus dificultades y posibles mejoras.

\section{Material y Métodos}

Estudio descriptivo de corte transversal. Acogiéndose a la Ley de Transparencia, se solicitó al CDE información sobre el proceso de mediación en salud entre los años 2005 y $2017^{8}$, obteniéndose datos estadísticos generales, caracterización de las mediaciones y datos de las prestaciones que originaron los reclamos. Esta información fue complementada con la disponible en la página web del CDE, http://www.cde.cl/gestion_institucional/ estadisticas/.

Para el registro de los datos se usó planilla Microsoft Excel (Microsoft, Redmond, WA, USA). Para el análisis descriptivo, se usaron estadígrafos como frecuencias absolutas y relativas.

\section{Resultados}

\section{Datos estadísticos}

Entre los años 2005 y 2017 se recibieron 13.510 reclamos. Los reclamantes fueron mujeres en $64,1 \%$ de los casos. Los pacientes supuestamente dañados en estos reclamos fueron mujeres en $55,3 \%$ de los casos.

Las regiones con mayor cantidad de reclamos fueron: Metropolitana (procuradurías de Santiago $24,2 \%$ y San Miguel 16,1\%), Valparaíso (11,5\%) y Concepción $(9,7 \%)$.

\section{Caracterización de las mediaciones}

Del total de reclamos recibidos durante los años estudiados, 12.744 (94,3\%) resultaron admisibles para mediación, de los cuales, 11.920 lograron concluirse en el período estudiado. La mayoría de los casos concluidos $(87,4 \%)$ finalizaron por decisión de las partes. Entre los casos así finalizados, 8.191 terminaron sin acuerdo y 2.229 con acuerdo (Figura 1).

Entre las mediaciones que finalizaron con acuerdo, la distribución según tipo de reparación se puede observar en la Tabla 1.

La duración promedio de las mediaciones fue 68,3 días y, en su mayoría, no fueron necesarias más de tres sesiones para su término (años 20132017). En cuanto a los comparecientes al proceso, se informó que entre los años 2011 y 2017 solo $21,5 \%$ acudió con representante legal.

Los montos indemnizatorios fueron registrados desde 2010. El monto promedio recibido por paciente indemnizado en el período estudiado fue de $\$ 14.862 .088$.

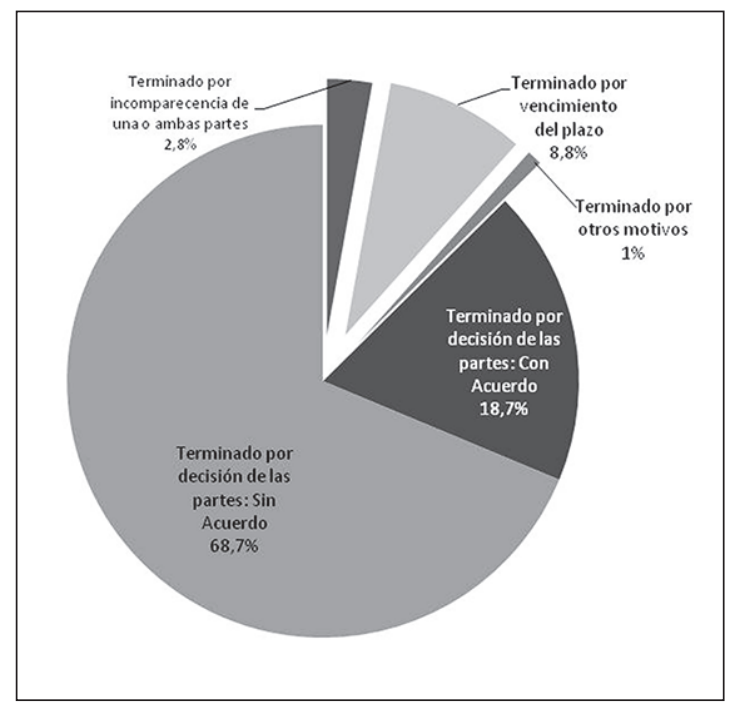

Figura 1. Causa de finalización de las mediaciones ante el Consejo de Defensa del Estado, durante el período estudiado (años 2005-2017). 


\section{Tabla 1. Tipo de acuerdo alcanzado en mediaciones terminadas "Con acuerdo entre las partes" (años 2005-2017)}

\begin{tabular}{|lrr|}
\hline Tipo de acuerdos alcanzados & Acuerdos & \% \\
\hline Indemnización & 595 & 26,7 \\
\hline Prestaciones asistenciales & 590 & 26,5 \\
\hline Disculpas o explicación de los hechos & 385 & 17,3 \\
\hline Más de un tipo de las antes mencionadas & 505 & 22,7 \\
\hline Acuerdo fuera de la mediación & 2 & 0,1 \\
\hline No especificado & 152 & 6,8 \\
\hline Total & 2.229 & 100,0 \\
\hline
\end{tabular}

Datos de la prestación que origina el reclamo

No se obtuvo la información sobre el tipo de prestador reclamado para los años 2008 y 2009. Para el resto del lapso, los prestadores más reclamados fueron: hospitales $(86,2 \%)$, consultorios de atención primaria $(7,8 \%)$, y servicios de salud $(0,05 \%)$. El 4,8\% fueron reclamos dirigidos a más de un prestador.

Los daños reclamados fueron, en su mayoría, invalidez parcial (33,2\%) (Figura 2).

Los incidentes que originaron reclamos empezaron a registrarse a partir del año 2012 y no se entregó el detalle anual. Su categorización puede observarse en la Tabla 2. Cabe destacar que puede haber más de un incidente que origine un reclamo.

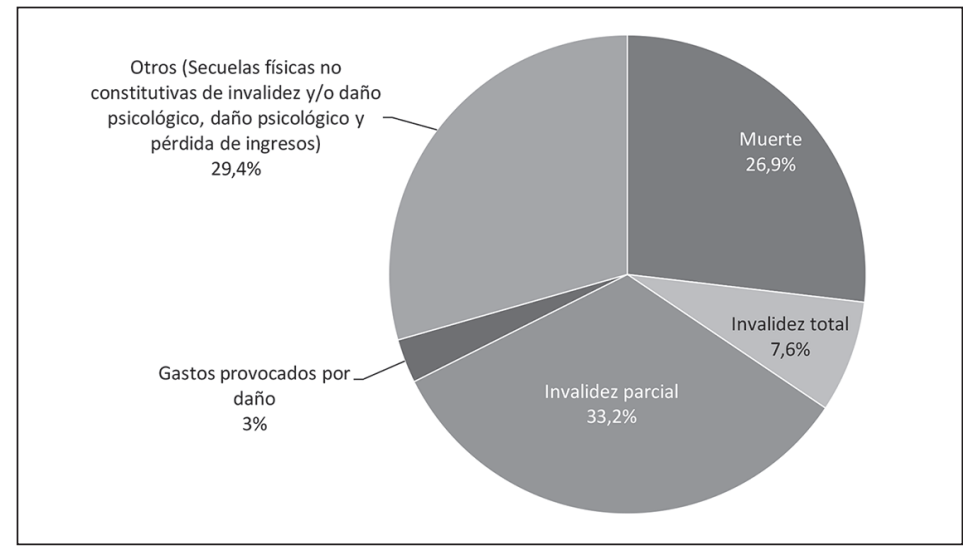

Figura 2. Daños alegados que originan el reclamo ante el Consejo de Defensa del Estado (años 2012-2017).

Tabla 2. Incidentes que originaron los reclamos ante el Consejo de Defensa del Estado (años 2012-2017)

\begin{tabular}{|lcc|}
\hline Incidente que origina el reclamo & Reclamos & \% \\
\hline Retardo en la atención & 2.458 & 23,6 \\
\hline Error de diagnóstico & 2.392 & 23,0 \\
\hline Error en la realización de procedimientos quirúrgicos o de enfermería & 1.974 & 19,0 \\
Error en la atención obstétrica & 917 & 8,8 \\
\hline Deficiencia en la atención de urgencia & 624 & 6,0 \\
Falta o deficiencia en la información entregada al paciente & 606 & 5,8 \\
Seguridad del paciente dentro del recinto hospitalario & 495 & 4,8 \\
\hline Errores en la utilización o retardo en la entrega de medicamentos & 348 & 3,3 \\
Seguridad y oportunidad en traslados y derivaciones de los pacientes & 224 & 2,2 \\
Ley de Derechos y Deberes del Paciente & 196 & 1,9 \\
Continuidad, pertinencia o especialización de quien brinda la atención & 62 & 0,6 \\
\hline Otros & 113 & 1,1 \\
\hline Total & 10.409 & 100,0 \\
\hline
\end{tabular}


El dato de la especialidad médica involucrada empezó a registrarse en el año 2010. Estas fueron ginecobstetricia $(22,4 \%)$, cirugía general $(21,5 \%)$, medicina interna $(21,1 \%)$, traumatología $(14,4 \%)$, pediatría $(11,5 \%)$, oftalmología $(5 \%)$ y neurocirugía $(4,2 \%)$. Los reclamos contra odontólogos fueron 168 (2,6\% del total de reclamos), mientras que otras del área salud (enfermería, laboratorio clínico y otras) suman un total de 199 (2,7\%).

\section{Limitaciones del estudio}

Escasa disponibilidad y estandarización de la información: los datos disponibles en la página web del CDE no estaban categorizados de la misma manera en todos los informes anuales, haciendo imposible su comparación estricta.

\section{Discusión}

\section{Montos de indemnización}

El Ministerio de Salud establece montos máximos a pagar por los prestadores públicos en virtud del procedimiento de mediación ${ }^{9}$. Dichos montos dependen del daño sufrido por el reclamante. La indemnización máxima por muerte es 3.500 U.F. ( $\$ 96.575 .000$ pesos chilenos aproximadamente), mientras que, según un estudio del año 2019, los montos indemnizatorios tras un juicio civil pueden elevarse a $\$ 520.000 .000^{10}$. El establecimiento de montos máximos de indemnización podría desincentivar al reclamante para llegar a un acuerdo extrajudicial.

El monto acordado en mediación es asumido por el prestador, menguando el presupuesto del Servicio al que pertenece, lo que va en desmedro de la entrega de atención de salud de calidad a sus usuarios, podría retardar la implementación de medidas correctivas o, incluso, fomentar la persecución judicial al profesional involucrado.

\section{Prestador donde se origina el reclamo}

$\mathrm{Al}$ año 2017, 76,87\% de la población chilena era beneficiaria de FONASA ${ }^{11}$. Sin embargo, hay que considerar que la atención primaria en salud (APS) presenta distintos mecanismos de administración y financiamiento, entre los cuales se cuentan las corporaciones municipales, que funcionan en 15,6\% del total de municipios del país, y los establecimientos administrados por organizaciones no gubernamentales ${ }^{12,13}$. Ambos tipos de organizaciones son personas jurídicas de derecho privado, y sus beneficiarios deben solicitar mediación ante la SIS, con los costos económicos que ello implica, dificultando el acceso al proceso $\mathrm{y}$ acentuando las desigualdades en salud.

En el presente estudio observamos que solo $6,3 \%$ de las mediaciones se originaron en una atención en APS. Es plausible pensar que este porcentaje subestime la realidad de los conflictos sanitarios originados ahí, y, por ende, muchos pacientes FONASA podrían no estar resultando beneficiados del proceso gratuito de mediación ante el CDE.

\section{Especialidades médicas involucradas}

Las tres especialidades más reclamadas son ginecobstetricia, medicina interna y traumatología. Esta tendencia es similar a la encontrada en otras instancias de reclamación en Chile: entre las pericias por responsabilidad médica ingresadas al Servicio Médico Legal, las especialidades más reclamadas fueron ginecobstetricia, cirugía y medicina interna ${ }^{14}$. Por otra parte, estudios sobre fallos de la Corte Suprema en casos de responsabilidad médica muestran que las especialidades más demandadas fueron ginecobstetricia, cirugía, medicina interna y traumatología ${ }^{10,15}$.

\section{Contexto internacional}

La mediación en salud tiene como referente a México: en 1996 nace la Comisión Nacional de Arbitraje Médico (CONAMED), organismo especializado de naturaleza ejecutiva, con el objeto de formular opiniones técnicas y recomendaciones tendientes a mejorar la atención médica. A diferencia del proceso de mediación chileno, el de CONAMED es vinculante, es decir, las partes están obligadas a respetar y cumplir sus fallos. Por otra parte, no solo busca la resolución de conflictos, sino también la contención de la medicina defensiva y la prevención de conflictos médicos mediante la retroalimentación a los prestadores de salud con la experiencia de los problemas ocurridos ${ }^{16}$.

\section{Posibles mejoras al proceso}

Creemos que la legislación chilena debería asegurar igualdad de trato para todos los usuarios en todas las instancias de su atención de salud, incluyendo un eventual reclamo, sin importar el régimen de salud al que pertenezcan.

Sería recomendable que se implementara un 
proceso institucional en el que los datos recabados durante las mediaciones se usaran para retroalimentar a los prestadores, de modo de poder concentrar los esfuerzos preventivos en aquellos procedimientos y especialidades que originan más conflictos en la atención, contribuyendo a la mejora continua del sistema de salud y traduciéndose en políticas públicas basadas en evidencia, mejor distribución de recursos en salud y un importante impacto a nivel social.

Finalmente, sería deseable contar con mediadores que tengan no solo habilidades en mediación, sino que fueran profesionales de la salud, pues son ellos quienes entienden mejor las complejidades de la relación médico-paciente.

Agradecimientos: A la Dra. Pamela Bórquez Vera, médico legista del Servicio Médico Legal (Santiago, Chile), por su contribución y revisión crítica del presente trabajo.

\section{Referencias}

1. World Health Organization, 2009. Marco Conceptual de la Clasificación Internacional para la Seguridad del Paciente Versión 1.1. Informe Técnico Definitivo. Disponible en https:/www.who.int/patientsafety/implementation/icps/icps_full_report_es.pdf [Consultado el 20 de agosto de 2018].

2. Gracia D. Prólogo. En: Beauchamp T, Childress JF. Principios de Ética Biomédica. Barcelona: Masson; 1999.

3. Hurtado-Hoyo E, Gutiérrez Zaldívar H, Iraola N, Rodríguez A. El síndrome clínico judicial. Rev Asoc Med Argent 2006; 119: 8-16.

4. Lagos M. Impacto de la mediación en la judicialización de conflictos jurídicamente relevantes: un análisis a base de la experiencia chilena de mediación por daños en salud. Revista de Estudios de la Justicia 2013; 18: 83-110.

5. Ley 19.966 Establece un Régimen de Garantías en Salud. Publicada 03 de septiembre del 2004. Disponible en www.leychile.cl [Consultado el 20 de agosto del 2018].

6. Decreto Ley 2763. Publicado el 03 de agosto de 1979 Reorganiza el Ministerio de Salud y crea los Servicios de Salud, el Fondo Nacional de Salud, el Instituto de Salud
Pública de Chile y la Central de Abastecimiento del Sistema Nacional de Servicios de Salud. Disponible en www.leychile.cl [Consultado el 20 de agosto del 2018].

7. Servicio de Mediación por Daños en Salud, Consejo de Defensa del Estado, Gobierno de Chile. Disponible en http://www.cde.cl/mediacion/ [Consultado el 20 de agosto de 2018].

8. Ley 20.285 Sobre Acceso a la Información Pública. Publicada el 20 de agosto del 2008. Disponible en www. leychile.cl [Consultado el 20 de agosto de 2018].

9. Resolución Exenta 142 del Ministerio de Salud. Publicada el 08 de abril del 2005. Establece montos máximos a pagar por los prestadores institucionales públicos en virtud del procedimiento de mediación establecido por la Ley no 19.966. Disponible en www.leychile.cl [Consultado el 20 de agosto de 2018].

10. Bravo L, Lagos D. Responsabilidad Médica en Chile: Fallos de la Corte Suprema de Justicia 2017. Int J Odontostomat 2019; 13 (3): 367-73.

11. Cuenta Pública Superintendencia de Salud. Gestión marzo 2014- marzo 2018. Disponible en http://www. supersalud.gob.cl/664/articles-16771_recurso_2.pdf [Consultado el 23 de enero de 2019].

12. Salud Municipal en Perspectiva. Asociación de Municipalidades de Chile AMUCH. Disponible en https:// www.amuch.cl/pdf/estudio_salud_municipal.pdf [Consultado el 23 de enero de 2019].

13. Eje Gestión de Recursos Financieros en Atención Primaria. Subsecretaria de Redes Asistenciales, División de Atención Primaria. Disponible en https://www.minsal. cl/wp-content/uploads/2016/09/4_GESTION-RECURSOS-FINANCIEROS-APS.pdf [Consultado el 23 de enero del 2019].

14. Moscoso K, Smok P. Responsabilidad sanitaria en odontólogos: Casuística de casos evaluados en el Servicio Médico Legal de Chile. Rev Med Chile 2015; 143 (3): 345-51.

15. Raineri G, Bravo L, Lagos D, Ciocca L. Análisis de los fallos de la Corte Suprema de Justicia de Chile en casos de responsabilidad médica, años 2014 y 2015. Rev Esp Med Legal Article in Press 10.1016/j.reml.2018.06.004.

16. Barría P, Saint George C. El modelo mexicano de arbitraje médico: Un aporte a la calidad de la medicina ya la comunicación entre médicos y pacientes. Revista de Derecho 2007; 17: 151-78. 